Instituto Internacional de Investigación y Desarrollo Tecnológico Educativo INDTEC, C.A.

DOI: https://doi.org/10.29394/Scientific.issn.2542-2987.2018.3.8.20.361-372

OAI-PMH: http://www.indteca.com/ojs/index.php/Revista Scientific/oai

\title{
La Tecnología y su Incidencia en la Sociedad del Conocimiento en la Edad Escolar
}

\author{
Autora: Tania Margarita Martínez de Padrón \\ Universidad Latinoamericana y del Caribe, ULAC \\ tania.martinez10@gmail.com \\ Caracas, Venezuela
}

\section{Resumen}

El ensayo a presentar basa su contenido en el impacto que está ocasionando la utilización de la tecnología de la información y la comunicación en la sociedad del conocimiento en edad escolar, específicamente en la educación Primaria Venezolana. Se destaca en el desarrollo del mismo, la participación del docente ante la utilidad de estos medios y la orientación que este brinda a los escolares. Asimismo, se destacan las políticas públicas en materia de TIC y educación que brinda el Gobierno Venezolano, la tenencia de las TIC en los hogares y el impacto de las nuevas tecnologías en el quehacer diario de los niños, niñas y adolescentes de la educación primaria. A lo largo del mismo, se especifican las condiciones para poder desarrollar un aprendizaje tecnológico significativo y su vinculación con el logro de una inteligencia colectiva. Cabe destacar, que el ensayo vislumbra la situación frente a las mencionadas Tecnologías y los retos a los que se enfrenta el docente para el aprovechamiento en el aprendizaje y facilitar que sea bien denominado en los escolares la sociedad del conocimiento.

Palabras clave: tecnología; sistema social; conocimiento. 


\title{
The Technology and its Incidence in the Society of Knowledge in School Age
}

\begin{abstract}
The essay to present bases its content on the impact that is causing the use of information technology and communication in the knowledge society of school age, specifically in the Venezuelan Primary Education. It is emphasized in the development of the same, the participation of the teacher before the utility of these means and the orientation that this one offers to the students. Likewise, the public policies on ICT and education offered by the Venezuelan Government, the possession of ICT in homes and the impact of new technologies on the daily work of children and adolescents in primary education are highlighted. Along the same, the conditions are specified to be able to develop a significant technological learning and its connection with the achievement of a collective intelligence. It is worth noting that the essay sees the situation in relation to the aforementioned technologies and the challenges faced by the teacher to make the most of learning and to make the knowledge society well-known among school children.
\end{abstract}

Keywords: technology; social system; knowledge. 


\section{Introducción}

La tecnología de la información y la comunicación (TIC), con el auge que obtuvo a partir de los años setenta se comienza una revolución en este ámbito que no tiene regresión y que ha provocado que todas las sociedades de distintas edades cambiaran sus formas de vida por una en donde las TIC tuvieran un papel sobresaliente frente a las relaciones humanas y en la educación.

Al respecto, destaca Meirinhos y Osório (2011): que "está comenzando una nueva etapa en la historia del hombre, donde por medio de su inteligencia comenzará a desarrollar tecnologías más sofisticadas y útiles para el beneficio de una sociedad en constante cambio y evolución" (pág. 35).

En relación con lo anterior, en Venezuela al igual que en el resto del mundo, se han enfrentado distintos tipos de brechas para poder incorporar las TIC a su modo de vida, adaptarse conforme avanzan estas para lograr una inteligencia colectiva, avanzando a una sociedad del conocimiento por medio de las Tecnologías, específicamente en la educación primaria.

Al respecto, el Ministerio del Poder Popular para la Educación, está dando pasos agigantados para que los escolares estén a la par con los nuevos cambios en materia de tecnología, al brindar a los estudiantes y docentes herramientas sofisticadas como las computadoras portátiles. A través de las cuales los actores educativos antes mencionados pueden intercambiar contenidos y hacer del acto educativo un verdadero aprendizaje tecnológico.

Después de estas consideraciones, se analizará durante el desarrollo como se está vivenciado el acto educativo ante las herramientas tecnológicas y el rol del docente ante la sociedad actual que se está formando en los centros educativos venezolanos, que basan su día a día en las redes del internet.

\section{Desarrollo}

Las herramientas tecnológicas y sus aligerados cambios han promovido 
la visión de nuevos patrones de pensamiento y nuevos grupos sociales, entre estos, los que de acuerdo con Prensky (2010): se podrían llamar nativos digitales, siendo estos una nueva generación de personas que posee el conocimiento tecnológico, que ha desarrollado nuevas conductas y que está destinada a efectuar un cambio impactante dentro de las organizaciones a las cuales ofrecen y prestan sus servicios.

De acuerdo con el planteamiento anterior, el autor antes citado, sostiene que la sociedad en formación en todo el mundo cuenta con miles de horas acumuladas detrás de un computador, interactuando a través de videojuegos, videochats, correos electrónicos, y en las distintas redes sociales del Internet. Para esta generación asimilar información y tomar decisiones les resulta tarea rápida, así como procesar informaciones paralelas.

Al hacer referencia al grupo social de décadas anteriores, encontramos a los mayores de 30 años denominados por el autor antes mencionado, "inmigrantes digitales", los cuales han aprendido a manejar la información, pero no han crecido con ella. Por tanto, este grupo es quien demuestra desinterés en incorporar las mismas en el quehacer diario, especialmente en su rutina de trabajo como en el caso de la profesión docente.

En concordancia, se destaca que a través de las visitas realizadas a algunos centros de educación primaria en Venezuela en especial en el estado Miranda, la autora de este ensayo puede dar fe que la labor de los docentes está lejos de la implementación en las aulas de las herramientas tecnológicas y tampoco existe la promoción entre los escolares para que adquieran conocimiento de los contenidos que imparten. A este particular, Torres (2012a):

Enfatiza que es importante reseñar que la mayoría de la población venezolana de cualquier edad tiene contacto y acceso a una computadora y al internet. Siendo estos dos recursos parte fundamental e importante para poder estar integrados en una sociedad dinámica, informada y 
comunicada, bien llamada la sociedad del conocimiento. (pág.

75).

No obstante, se observan a estas generaciones, especialmente docentes, que permanecen bajo un viejo paradigma de continuar haciendo más de lo mismo y mantenerse alejados de la innovación, dejando de poner en práctica, la creación de blogs, foros y otras estrategias tecnológicas de punta, solo presentan a sus escolares, la sociedad del futuro, pizarra y cuadernos.

En este orden de ideas, es preciso señalar que en Venezuela según el autor antes señalado, la población más activa en la rutina de las Tic, es la de edad escolar, considerando que esa población es la que está en mayor uso motivado a que en esa edad se asiste a alguna institución educativa y por lo tanto tienen la necesidad de utilizar esas herramientas y es la población más fácil de adaptarse a las tecnologías, sin embargo no se cuenta con la orientación del docente para que el escolar incursione en este vertiginoso mundo.

Lo antes dicho, enfatiza el mismo autor, que "en Venezuela el uso que los cibernautas en edad escolar le dan al internet, es acceder a redes sociales, descargar música y casi en últimos lugares se encuentran el acceder a la instrucción continua, visitar foros especializados" (Torres, 2012b, pág. 86).

Es de destacar, que sobre todo la población joven, utiliza el internet principalmente como un medio de distracción y diversión, y poco es utilizado como fuente de investigación, o con uso didáctico y educativo. Es por ello que es imperante la participación del docente, motivado a que de acuerdo con Tadesco (2011:41): esta concentración de conocimientos e informaciones en los circuitos de las nuevas tecnologías como es el caso de internet, explica la necesidad de incorporar adecuadamente la dimensión tecnológica en las políticas educativas democráticas. Continúa agregando que no hacerlo puede 
condenar a la marginalidad a todos los que queden fuera del dominio de los códigos que permitan manejar estos instrumentos.

Es claro que el uso de las Tic, sobre todo en la población más joven está basado en la poca orientación que le brinda la escuela, si esta, desde que el niño entra en los centros educativos fuese dirigida en este sentido, los escolares pudiesen apoderarse de ella de manera razonable en pro de su formación.

En Venezuela de acuerdo con Morales (2015): es cada vez más grande el uso de las TIC, pero también es cierto que en el país existe falta de orientación en los escolares que no permite que se aproveche la tecnología para lo que fueron creadas y esa desinformación es motivada al bajo nivel de utilización que le asignan los profesores a la misma, es imperante una facilitación masiva al gremio para que esta sea llevada a las aulas y sea aprovechada para alcanzar el aprendizaje significativo en el ámbito tecnológico.

Es preciso señalar, que entre las políticas educativas del Gobierno Venezolano en torno a la cobertura y el uso de las TIC en el ámbito educativo, es lograr que toda la población paulatinamente vaya teniendo acceso a las Tecnologías, comenzando con los programas en donde todas las escuelas desde nivel inicial deben estar equipadas con computadoras e internet. Es por ello que se ha asignado una computadora portátil a los niños, adolescentes y a los docentes, con la intención que exista un intercambio positivo entre estos actores.

Sin embargo, esto ha sido considerado una problemática, motivado a que no se adiestra al profesorado para que implemente este recurso tan valioso en las aulas constantemente, quedando este, como inapropiado para el docente, ya que no encuentra motivación para planificar estrategias e impartir contenidos para el aprendizaje de los niños y adolescentes.

Es preciso hacer referencia a la llamada sociedad del conocimiento, que 
es referida a la capacidad de preparación de los ciudadanos, al desarrollo de sus posibilidades de investigación e innovación y de creatividad adecuada para procesar informaciones y conocimientos. En tal sentido, se puede diferenciar que sociedad de la información no es igual a sociedad del conocimiento.

Es la sociedad de la información el primer concepto que se desarrollara en los años setenta y ochenta. Considerando a la sociedad de la información exclusivamente como consumidora de información. Será con el despegue del internet y las innovaciones tecnológicas que se comienza a tener un efecto evidente en las sociedades. (Palomares, 2012, pág. 89).

Asimismo, destaca la autora que la sociedad del conocimiento se desarrolla hacia finales de los años noventa cuando el despegue tecnológico es irremediable y los alcances de la tecnología comienzan a sobrepasar lo imaginado.

Al respecto, la sociedad del conocimiento es una sociedad que se distingue por consumir, producir y distribuir información y conocimiento. Así pues, el avance tecnológico es el motor que logra cambiar a una sociedad solamente informada por una sociedad productora, que está en mayor contacto con las Tecnologías y las usan con la finalidad de crear redes de inteligencia colectiva.

En concordancia con lo anterior, Marqués, (citado en Ferreira, 2016a): expresa que la sociedad de la información es la base de la sociedad del conocimiento, ya que la sociedad de la información incluye la dimensión social, cultural, política, económica e institucional. Es preferible el concepto de sociedad del conocimiento al de sociedad de la información, porque esta captura mejor la complejidad y el dinamismo de los cambios que se dan en la actualidad.

Sobre la base de lo antes expuesto, ambos conceptos están relacionados y describen el comportamiento de la sociedad en la que vivimos. 
Una sociedad llena de información, pero que ha aprendido a relacionarse y crear propios conocimientos para dejar de ser solo espectador para convertirse en un sujeto activo.

A tal planteamiento se aúna lo descrito por Sánchez (2010a, pág. 3): cuando se refiere a que "la aprehensión tecnológica de los niños se desarrolla de modo natural, intuitivo y libre, asimismo destaca que la intensa emergencia tecnológica que caracterizará el desarrollo de la Sociedad del Conocimiento exige mentes muy abiertas, intuitivas, flexibles y desapegadas de lo conocido, y en este contexto los niños cuentan con ventajas asociadas a su proceso de crecimiento y aprendizaje.

Es de esta forma como a través de la Sociedad del conocimiento, se podrá lograr en mayor escala la inteligencia colectiva logrando comunicar y crear redes educativas donde todos los seres humanos sean capaces de compartir y crear conocimiento a partir de la realidad que cada uno viva, y de sus distintas experiencias y saberes que solamente ellos saben y comparten para hacer un conocimiento más rico, profundo, productivo, variado, y sobre todo significativo.

Sobre esta base, es importante destacar lo descrito por Sánchez (2010b), respecto a:

La presencia de los niños y adolescentes en las redes sociales promueve la información compartida y el desarrollo de una función colaborativa y cooperativa que está transformando los tradicionales modelos sociales de relaciones basadas en los instrumentos institucionales. Las redes nos comunican, y la comunicación colectiva nos ayuda a poner en común nuestros problemas y a buscar fórmulas colectivas de desarrollo. Ésta es otra de las tendencias de la Sociedad del Conocimiento que se están extendiendo de forma veloz entre la población infantil. Una vez que se consolida la comunicación estable y plural surgen las experiencias de compartición y desarrollo cooperativo (pág. 5).

Es por lo antes planteado que es de suma importancia la labor del 
docente de educación primaria en la orientación educativa de estos medios, ya que el conocimiento y el pensamiento son actos que se realizan de manera colectiva y no aislada, pues será con una sociedad del conocimiento que se podrá hacer un uso adecuado y responsable de las Tecnologías siempre que estas estén correctamente direccionadas hacia el aprendizaje.

\section{Conclusión}

Pensando ahora en incorporar las Tecnologías a las sociedades del conocimiento en edad escolar, se puede tomar en cuenta lo descrito por Majfud (2008): quien expone que "la educación es una especialidad de la cultura: su función es el desarrollo humano en una determinada área que incluye la seguridad física y psicológica, el desarrollo económico, el desarrollo de la experiencia existencial a través del arte". (pág. 1).

Es por ello que sería un gran reto si se desarrollan planes y programas pensados en el contexto actual de los venezolanos, sobre todo en el contexto de los escolares actuales, si se lleva a cabo sin copiar modelos ajenos a nuestra realidad, se podrá hablar de autenticidad tecnológica. Cabe destacar, que las TIC fueron creadas para simplificar la vida de los alumnos y abrirle múltiples posibilidades para hacer significativa su educación.

Hoy día, ya es evidente que la sociedad en edad escolar está íntimamente ligada con el ámbito tecnológico, dominando con mucha destreza estas herramientas, por ende, los docentes deben entender que el cambio de paradigma es oportuno y necesario.

Sobre esta base, se debe pensar en la transformación de las estrategias del docente de una manera distinta, comenzando desde la capacitación universitaria, introduciendo en el pensum de estudio de la carrera, asignaturas que tengan que ver directamente con la inclusión de la tecnología y el internet

dentro del aula y cambiar el panorama pedagógico en la educación primaria venezolana. 
Es de aseverar, en concordancia con Ferreira (2016b): que el docente al emplear estrategias tecnológicas en la enseñanza de cualquier materia o habilidad creativa, resultará significante a la sociedad del conocimiento en edad escolar, y en particular, mediante Internet, aplicando las técnicas adecuadas, se podrán evidenciar cambios nunca antes visto de parte de los escolares en su desempeño dentro de los centros educativos y su rendimiento.

A este particular, debe existir una sensibilización ante los entes superiores para que de manera conjunta con el gobierno comience a darse la transformación en el área tecnológica pedagógica. Así las sociedades del conocimiento con todos sus cúmulos de creatividad y capacidades de inteligencia jugaran un papel preponderante en la educación, pues se logrará una educación permanente, actualizada y significativa.

Para concluir, es necesario insistir ante la transformación del ejercicio de la profesión docente, formar educadores que eduquen un verdadero ciudadano del futuro, el cual se vislumbra con herramientas cada día más sofisticadas, ante las cuales la figura del maestro no debe desaparecer. Este debe continuar siendo el guiador y orientador de la sociedad del conocimiento en edad escolar. Sobre esta base, se busca pensar y mirar a las Tecnologías y la Sociedad del conocimiento en edad escolar desde una sola perspectiva, la cual es conseguir que los individuos se integren en sociedades del conocimiento de acuerdo con las herramientas innovadoras que se van presentando.

\section{Referencias}

Ferreira, E. (2016a,b). La formación del Docente del Siglo XXI. Centro de Información INAFOCAM. República Dominicana. Recuperado de: http://planlea.listindiario.com/2016/09/la-formacion-del-docente-delsiglo-xxi 
Meirinhos, M., \& Osório, A. (2011). Formación y Aprendizaje en la Sociedad de la Información: Elementos de un nuevo Paradigma. Instituto Politécnico de Bragança, Biblioteca Digital. Braganza, Portugal: Editora Hybryd Days. Recuperado de: http://hdl.handle.net/10198/6959

Majfud, J. (2008). La inteligencia colectiva. Revista Iberoamericana de Educación, 45(2). ISSN: 1681-5653. Edita: Organización de Estados Iberoamericanos para la Educación, la Ciencia y la Cultura (OEI).

Morales, N. (2015). Tecnologías de Información y Comunicación para aprender y colaborar. México: Editorial Trillas.

Prensky, M. (2010). Nativos e Inmigrantes Digitales. Depósito legal: M24433-2010. Institución Educativa SEK. Cuadernos SEK 2.0. Edita: Distribuidora SEK, S.A., Impresión: Albatros, S.L. Adaptación al castellano del texto original "Digital Natives, Digital Immigrants". Recuperado de: https://www.marcprensky.com/writing/PrenskyNATIVOS\%20E\%20INMIGRANTES\%20DIGITALES\%20(SEK).pdf

Palomares, I. (2012). La Sociedad de la Información y el Conocimiento. México: Universidad Nacional Autónoma de México.

Sánchez, J. (2010a,b). Nuevos Escenarios de Participación Infantil en la Sociedad del Conocimiento. III Congreso Internacional Solidario. (UNICEF). España: Gijón.

Tadesco, J. (2011). Los Desafíos de la Educación Básica en el Siglo XXI. Revista Ibero-Americana de Educación, N.55, págs. 31-47.

Torres, R. (2012a,b). Sociedad de la información y Sociedad del conocimiento. Mérida, Venezuela: Universidad de los Andes, ULA. 


\section{Tania Margarita Martínez de Padrón \\ e-mail: tania.martinez10@gmail.com}

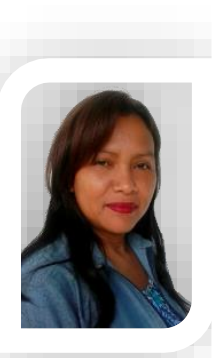

Nacida en Venezuela. Licenciada en Educación egresada de la Universidad Nacional Experimental Simón Rodríguez (UNERS). Especialista en Planificación y Evaluación (UNERS). Diplomado en Investigación. Universidad Nacional Experimental de la Fuerza Armada Nacional Bolivariana (UNEFA). Magister Scientiarum en Tecnología Educativa. Universidad Nacional Experimental de la Fuerza Armada Nacional Bolivariana (UNEFA). Doctorante en Ciencias de la Educación Universidad Latinoamericana y del Caribe (ULAC). Subdirectora y Docente de Aula en ejercicio de la: U.E.E. "La cruz". Docente de Postgrado de la Universidad Nacional Experimental de la Fuerza Armada Nacional Bolivariana (UNEFA). Tutora de Tesis de Postgrado (UNEFA).

El contenido de este manuscrito se difunde bajo una Licencia de Creative Commons ReconocimientoNoComercial-Compartirlgual 4.0 Internacional 